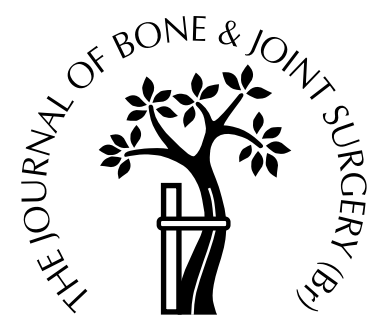

\title{
Interobserver and intraobserver variation in classification systems for fractures of the distal humerus
}

\author{
A. M. Wainwright, J. R. Williams, A. J. Carr \\ From the John Radcliffe Hospital and Nuffield Orthopaedic Centre, Oxford, England
}

$\mathbf{W}$ e assessed the inter- and intraobserver variation in classification systems for fractures of the distal humerus. Three orthopaedic trauma consultants, three trauma registrars and three consultant musculoskeletal radiologists independently classified 33 sets of radiographs of such fractures on two occasions, each using three separate systems.

For interobserver variation, the Riseborough and Radin system produced 'moderate' agreement (kappa = 0.513), but half of the fractures were not classifiable by this system. For the complete AO system, agreement was 'fair' (kappa $=0.343$ ), but if only AO type and group or AO type alone was used, agreement improved to 'moderate' and 'substantial', respectively (kappa $=0.52$ and 0.66 ). Agreement for the system of Jupiter and Mehne was 'fair' $($ kappa $=0.295)$. Similar levels of intraobserver variation were found.

Systems of classification are useful in decision-making and evaluation of outcome only if there is agreement and consistency among observers. Our study casts doubt on these aspects of the systems currently available for fractures of the distal humerus.

J Bone Joint Surg [Br] 2000;82-B:636-42.

Received 25 November 1998 ; Accepted after revision 6 October 1999

J. R. Williams, DM, Senior Lecturer in Trauma and Orthopaedic Surgery

The Medical School, University of Newcastle, Newcastle-upon-Tyne NE2 4HH, UK.

A. M. Wainwright, FRCS, Specialist Registrar

A. J. Carr, ChM, Consultant Orthopaedic Surgeon

Nuffield Orthopaedic Centre, Windmill Road, Headington, Oxford OX3 7LD, UK.

Correspondence should be sent to $\mathrm{Mr} \mathrm{A}$. M. Wainwright at 30 Orchard Close, Thame, Oxfordshire OX9 3JR, UK.

(C)2000 British Editorial Society of Bone and Joint Surgery 0301-620X/00/59673 \$2.00

636
Systems for the classification of fractures are used in clinical practice and research to provide a language for communication, make decisions about treatment, evaluate the prognosis, and to report and compare results. Ideally, such a system should be reliable, reproducible, all-inclusive, mutually exclusive, logical and clinically useful. ${ }^{1}$

The distal humerus is a complex skeletal area to reconstruct, and several systems of classification have been proposed. The manner of treatment has changed markedly during the last 30 years from advising against operation ${ }^{2}$ to advocating open reduction and internal fixation. ${ }^{3,4}$ The systems have reflected these changes in the way in which fractures have been analysed.

Our aim was to assess the interobserver and intraobserver variability for classification of fractures of the distal humerus using three systems, namely, that of Riseborough and Radin, ${ }^{2}$ the AO group ${ }^{5}$ and Jupiter and Mehne. ${ }^{6}$ We did not attempt to assess how they assisted clinical management.

\section{Patients and Methods}

Nine assessors classified the anteroposterior (AP) and lateral radiographs of 33 patients with fractures of the distal humerus using the three systems. These radiographs were normal films selected at random. The assessors were three trauma registrars, three orthopaedic trauma consultants and three musculoskeletal consultant radiologists. The same assessors reclassified the same radiographs after at least three months. When the assessor was not able to classify the fracture within a system a separate category was added.

Classification systems (Figs 1 to 3). Each assessor was provided with diagrams illustrating and describing in detail the three systems of classification in use, taken from the initial descriptions by the original authors.

Riseborough and Radin $^{2}$ described a series of intercondylar $\mathrm{T}$-fractures and divided them into four types reflecting severity as follows:

Type I. There is no displacement of the fragments.

Type II. The trochlear and capitellar fragments are separated but not appreciably rotated in the frontal plane.

Type III. As for type II but the fragments are also significantly rotated. 


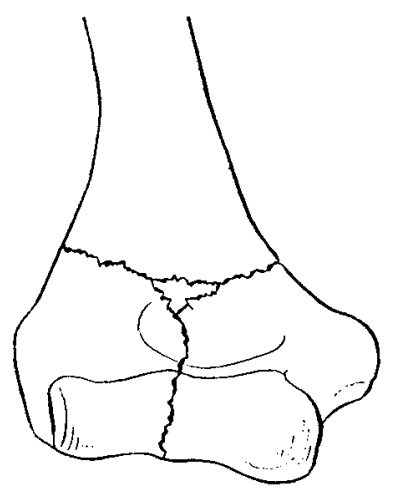

Type I. Undisplaced

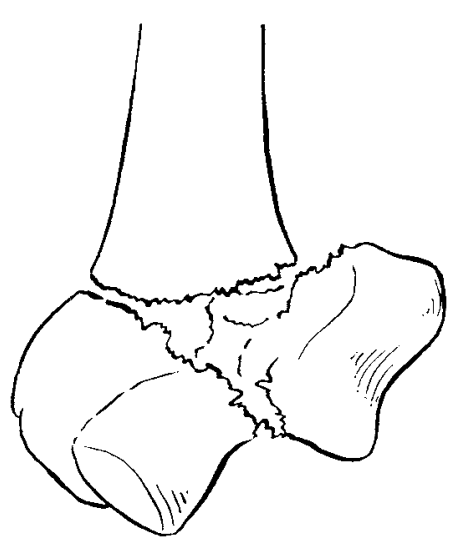

Type III. Separated and rotated

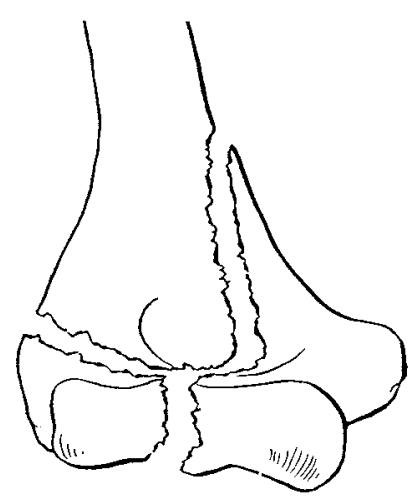

Type II. Separated fragments

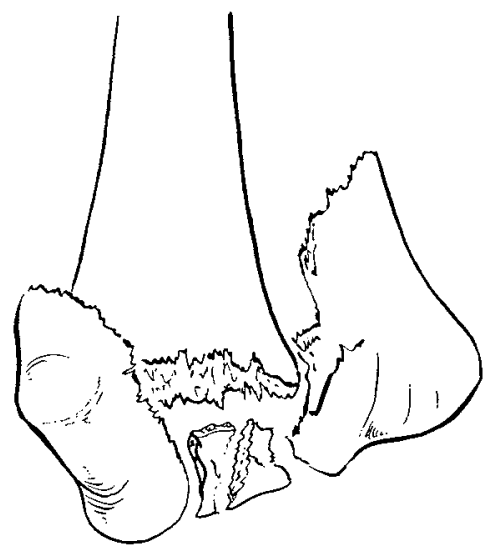

Type IV. Comminution and wide separation

Fig. 1

Summary of the classification of Riseborough and Radin of intercondylar T-fractures of the distal humerus ${ }^{2}$ (adapted with permission from Riseborough EJ, Radin EL. Intercondylar T fractures of the humerus in the adult: a comparison of operative and non-operative treatment in 29 cases. J Bone Joint Surg [Am] 1969;51-A:130-41).

Type IV. There is severe comminution of the articular surface and wide separation of the humeral condyles.

The AO classification ${ }^{5}$ arose from efforts to produce a comprehensive system for fractures of the long bones. The distal segment ' 13 ' of the humerus is divided into three types as follows:

Type A. Extra-articular fractures.

Type B. Partial articular fractures; these maintain some continuity between the shaft and the articular surface.

Type $C$. Complete articular fractures.

These are subdivided into groups 1, 2 and 3 on the basis of the location of the line of the fracture and the amount of fragmentation. They are then subdivided into subgroups $\bullet 1$, $\cdot 2$ and $\bullet 3$ by specific further qualifications.

Jupiter and Mehne ${ }^{6}$ developed a different comprehensive system based on the surgical anatomy and the techniques used for reconstruction. There are three basic categories as follows:
Grade I. Intra-articular fractures.

Grade II. Extra-articular, intracapsular fractures.

Grade III. Extracapsular fractures.

Intracapsular fractures are subdivided into: $\mathrm{A}$, single column; B, bicolumnar; C, capitellar; and D, trochlear.

The distance of the fracture from the joint and the direction of the transverse limb of the fracture determine further categories.

Statistical methods. It would be ideal to have an absolute standard with which to compare the observations but any study of this type is limited by our ability to define accurately the pattern of the fracture. Even if findings at the time of operation were taken as the standard, there may have been disagreement on classification at this stage, and not all fractures require an operation. Lacking an absolute standard the categories of classification were treated as nominal data and we used the kappa statistic for evaluation of inter- and intraobserver agreement. The kappa statistic is 


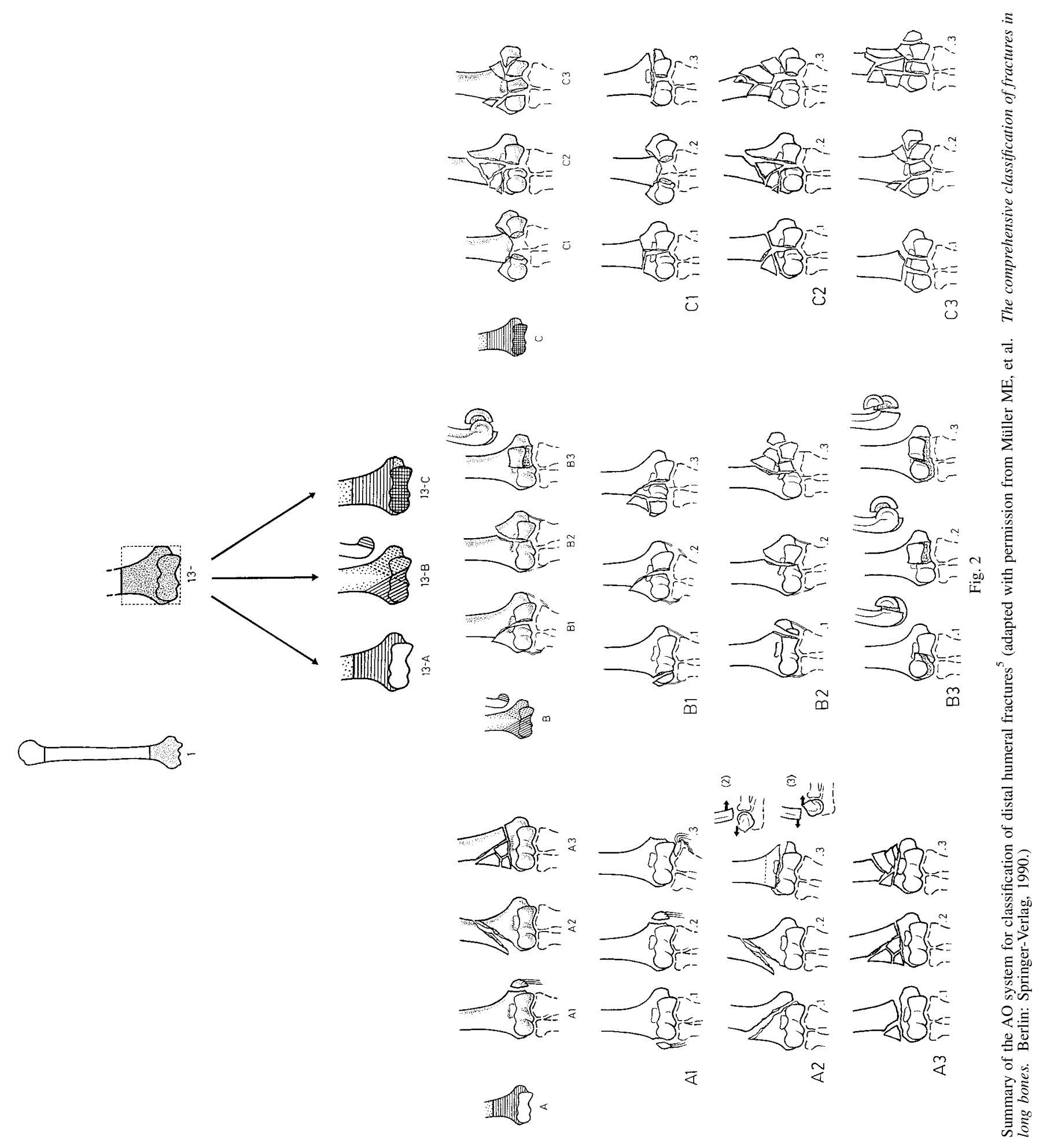




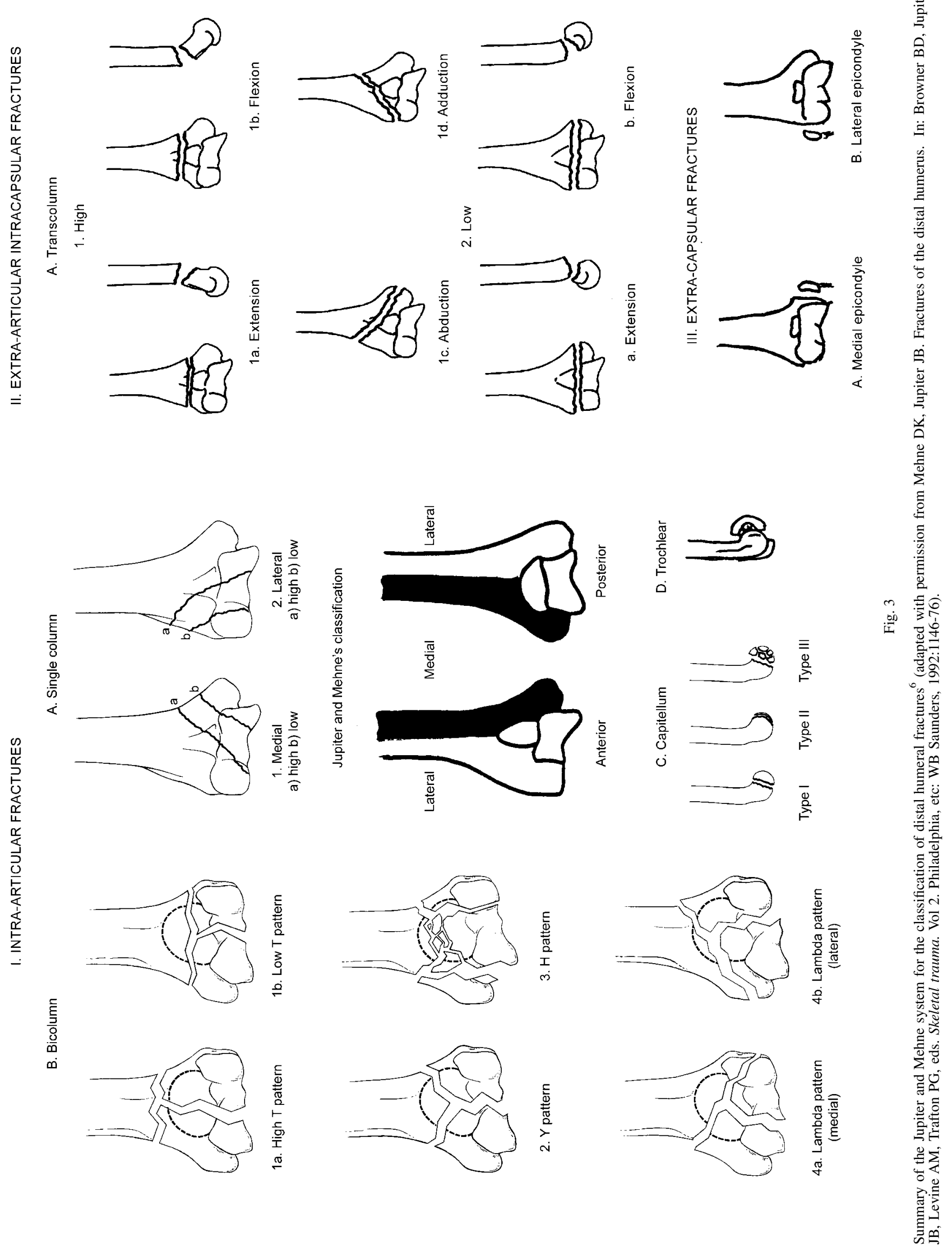

VOL. 82-B, No. 5, JULY 2000 
a chance corrected measure of agreement for nominal data, first described by Cohen. ${ }^{7}$ It compares an observed measure of agreement $\mathrm{P}(\mathrm{A})$, with the level of agreement expected by chance alone $\mathrm{P}(\mathrm{E}){ }^{8,9}$ The maximum value of 1.0 means that every assessor agrees with every other on every case. A value of 0 indicates no more agreement than expected by chance alone, and the value may be less than 0 . Interpretation of the kappa value was based on the guidelines proposed by Landis and Koch, ${ }^{10}$ as shown in Table I.

\section{Results}

All 33 sets of radiographs were classified by all nine assessors. In the Riseborough and Radin classification all the groups were used for at least one observation. In the AO classification all except two of the subgroups were used; the exceptions were A1.1 and A1.2. In the Jupiter and Mehne classification all except three categories, ID, IIIA and IIIB, were used.

Interobserver variation. The interobserver agreement for the classification systems is summarised in Table II. In $48 \%$ of the assessments, the fractures could not be classified using the Riseborough and Radin system and there was some disagreement as to which of the fractures could be classified using this method. At least one of the observers thought that a fracture could not be classified by the system in 24 out of the 33 cases. In only seven was there complete

Table I. Interpretation of the kappa statistic according to Landis and Koch ${ }^{10}$

\begin{tabular}{ll}
\hline Kappa statistic & Strength of agreement \\
\hline$<0.00$ & Poor \\
0.00 to 0.20 & Slight \\
0.21 to 0.40 & Fair \\
0.41 to 0.60 & Moderate \\
0.61 to 0.80 & Substantial \\
0.81 to 1.00 & Almost perfect \\
\hline
\end{tabular}

agreement among observers that the fracture could not be classified. Overall, the interobserver agreement was 'moderate' $($ kappa $=0.513)$.

The AO system has 27 subgroups and had 'fair' interobserver agreement (kappa=0.343). The agreement improved when assessment was by the nine AO groups (kappa $=0.521$, 'moderate'), or by the three AO types alone (kappa $=0.661$, 'substantial').

The Jupiter and Mehne system ${ }^{6}$ has 19 categories and gave an overall 'fair' agreement (kappa $=0.295$ ).

Intraobserver agreement. This showed a similar pattern to interobserver agreement (Table III). Only seven observers repeated the classification and one did not reclassify the AO subgroup.

The system of Riseborough and Radin ${ }^{2}$ produced wide ranges of intraobserver agreement (kappa scores between 0.364 and 0.696).

Agreement in the AO classification by subgroup was 'fair to moderate' (kappa $=0.254$ to 0.536). When classified by AO group agreement was 'moderate to substantial' (kappa $=0.466$ to 0.75 ), and by AO type was higher (kappa $=0.489$ to 0.835 ). Two observers were consistent in their intraobserver agreement for type (kappa > 0.81).

Using the Jupiter and Mehne system, the intraobserver agreement varied widely (kappa $=0.001$ to 0.550$)$. The lowest level occurred with one observer who favoured one class on the initial assessment and another on the subsequent occasion, putting almost half (15/33) of the readings into the IB 3 class and only one in the IB4b class on the first assessment, and then reversed this on the second.

\section{Discussion}

Fractures of the distal humerus account for $2 \%$ of all fractures and about one-third of those at the elbow. A review of the history of the classification of fractures of the elbow is given elsewhere. ${ }^{11}$

Table II. Kappa scores for interobserver agreement

\begin{tabular}{llllll}
\hline Observers & Riseborough and Radin & AO subgroup & AO group & AO type & Jupiter and Mehne \\
\hline Consultant trauma surgeons & 0.521 & 0.335 & 0.476 & 0.693 & 0.348 \\
Consultant radiologists & 0.518 & 0.320 & 0.618 & 0.802 & 0.262 \\
Registrars & 0.568 & 0.272 & 0.458 & 0.613 & 0.199 \\
Overall & 0.513 & 0.343 & 0.521 & 0.661 & 0.295 \\
\hline
\end{tabular}

Table III. Kappa scores for intraobserver agreement

\begin{tabular}{llllll}
\hline Observers & Riseborough and Radin & AO subgroup & AO group & AO type & Jupiter and Mehne \\
\hline A & 0.696 & 0.536 & 0.750 & 0.788 & 0.475 \\
B & 0.364 & 0.254 & 0.562 & 0.690 & 0.001 \\
C & 0.664 & 0.378 & 0.566 & 0.811 & 0.550 \\
D & 0.362 & 0.428 & 0.470 & 0.703 & 0.239 \\
E & 0.680 & 0.336 & 0.622 & 0.835 & 0.400 \\
F & 0.422 & 0.440 & 0.466 & 0.489 & 0.140 \\
G & 0.601 & 0.395 & 0.646 & 0.556 & 0.275 \\
Mean & 0.541 & & 0.583 & 0.695 & 0.297 \\
\hline
\end{tabular}


It has been proposed that there should be two types of system of classification, $^{12}$ one (taxonomy) for research purposes to make valid comparisons between groups, and the other (treatment) for clinical use to determine management and prognosis. For either of these an ideal system should be easy to use, widely accepted and produce good inter- and intraobserver agreement. ${ }^{1}$ Our aim was to assess the last and no attempt was made to comment on the ease of use or acceptability.

Although systems of classification are widely used, several studies have shown that many in current use do not have consistency among or within observers. For the Gustilo and Anderson classification of open fractures, Brumback and Jones ${ }^{13}$ found moderate to poor interobserver agreement in a survey of 245 orthopaedic surgeons. Previously, Horn and Rettig ${ }^{14}$ had shown moderate interobserver reliability.

Johnstone, Radford and Parnell ${ }^{15}$ assessed interobserver variation using the AO/ASIF classification of fractures of the long bones. They compared individual classification with a consensus view and found agreement of only $32 \%$. There was moderate agreement for the Evans classification of trochanteric fractures. ${ }^{16}$ Observers agreed on the stage in Garden's classification of fractures of the femoral neck in only $22 \%{ }^{17}$ Analysis of the interobserver reliability and intraobserver reproducibility in the classification of fractures of the distal radius found moderate agreement between observers for the Mayo and fair agreement for the Frykman, Melone and AO classifications. ${ }^{18}$ Another study showed only fair agreement of the AO system for the distal radius when assessed for the potential 27 subgroups. ${ }^{19}$

In a study of fractures of the proximal humerus there was fair or poor inter- and intraobserver reliability of classification using Neer's system and the AO system, ${ }^{20}$ and in another study there was moderate interobserver reliability, but higher intraobserver reproducibility of the Neer classification. ${ }^{21}$ These studies prompted an Editorial by Burstein $^{22}$ which recommended that the Neer classification system was unwarranted as a means of differentiating treatment and correlating outcomes in clinical series. He concluded that all studies using a classification scheme to assess clinical outcome should show that there had been acceptable intraobserver repeatability and interobserver reliability.

To our knowledge, there have been no other studies published of the interobserver and intraobserver reliability of the classification of fractures of the distal humerus. Johnstone et al $^{15}$ found that classification of a sole fracture of the distal humerus gave the greatest amount of variation from the consensus classification of all the fractures which they analysed.

It may be that the process of classification can only be accurately performed after the fracture has been assessed by direct vision at operation. However, part of the function of a useful system of classification is to determine the best method of treatment, so that preoperative planning can be performed before embarking on an operation.

In our study, observers commented that further radiographs with traction applied to the limbs are often made in the operating theatre. This may aid in classifying the fractures, but we did not assess this. It has also been suggested that CT may clarify the patterns of fracture. This theory has been investigated for the proximal humerus but two studies of the Neer classification system ${ }^{23,24}$ showed that $\mathrm{CT}$ added little to improving reproducibility for those fractures.

One reason why the agreement may be higher in our assessment compared with a general population is that the observers were part of a study and took special care. They were also from the same unit and had common experience of the patients in many cases. The order of presentation was not rerandomised, although there was a considerable period between reviews. Reasons for the lower agreements may be that the systems of classification were not clearly understood, or there was fatigue after reviewing many similar radiographs. Bias may have also been introduced by the fact that the assessors in our study were more familiar with the AO system. Only two stated that they would routinely use the AO system to describe a fracture, most preferring to describe the fracture in a sentence.

There are also intrinsic differences among the three systems. The AO system employs a stepwise classification, which becomes more detailed to use with each stage. The Riseborough and Radin method is a single assessment into four categories of increasing severity. The Jupiter and Mehne system has elements of both of these approaches. One observer commented that there appear to be two phases in classification, an initial interpretation of radiographs to produce a mental image of a fracture in three dimensions, and subsequently, a matching of the threedimensional image to the system of classification. When developing future systems it may be useful to analyse at which stage the agreement is lost.

Systems of classification are useful in decision-making and comparison of outcomes only if there is a high level of agreement among observers and consistency by them. Our study has cast doubt on these features of current systems for fractures of the distal humerus.

We found that the Radin and Riseborough classification was not applicable to half of these fractures and produced moderate agreement. There was only fair agreement in the AO and the Jupiter and Mehne systems, but when only the basic outline classification was used there was substantial agreement.

We would like to thank Mr Clasper, Mr Handley, Mr Kamborouglou, Mr Keys, Dr McNally, Mr Oliver, and Dr Ostlere for taking the time to classify these fractures. We are grateful to the authors and publishers of the three classification systems for allowing us to reproduce diagrams taken from their original texts. We would also like to thank the staff of the radiology and photographic departments and the Cairns and Girdlestone Memorial Libraries for their help.

No benefits in any form have been received or will be received from a commercial party related directly or indirectly to the subject of this article. 


\section{References}

1. Martin JS, Marsh JL. Current classification of fractures. Radiol Clin N Am 1997;35:491-506.

2. Riseborough EJ, Radin EL. Intercondylar T fractures of the humerus in the adult: a comparison of operative and non-operative treatment in 29 cases. J Bone Joint Surg [Am] 1969;51-A:130-41.

3. Holdsworth BJ, Mossad MM. Fractures of the adult distal humerus: elbow function after internal fixation. J Bone Joint Surg $[\mathrm{Br}]$ 1990;72-B:362-5.

4. Jupiter JB. Complex fractures of the distal part of the humerus and associated complications. J Bone Joint Surg [Am] 1994;76-A:1252-64.

5. Müller ME, et al. The comprehensive classification of fractures in long bones. Berlin: Springer-Verlag, 1990.

6. Jupiter JB, Mehne DK. Fractures of the distal humerus. Orthopedics 1992;15:825-33.

7. Cohen J. A coefficient of agreement for nominal scales. Educ Psychol Meas 1960;20:37-46

8. Posner KL, Sampson PD, Caplan RA, Ward RJ, Cheney FW. Measuring interrater reliability among multiple raters: an example of methods for nominal data. Stat Med 1990;9:1103-15

9. Seigel S, Castellan NJ. Measures of association and their tests of significance: nominally scaled data and the kappa statistic. Non parametric statistics for the behavioural sciences New York, etc: McGraw Hill, 1988:284-91.

10. Landis RJ, Koch GG. An application of the hierarchical kappa-type statistic in the assessment of majority agreement among multiple observers. Biometrics 1977;33:363-74.

11. Williams JR. The elbow, including humeral shaft, distal humerus and forearm. In: Pynsent PB, Fairbank JCT, Carr AJ, eds. Classification of musculoskeletal trauma. Oxford: Butterworth-Heinemann, 1999:131.

12. Bernstein J, Monaghan BA, Silber JS, DeLong WG. Taxonomy and treatment: a classification of fracture classifications. J Bone Joint Surg [Br] 1997;79-B:706-7.

13. Brumback RJ, Jones AL. Interobserver agreement in the classification of open fractures of the tibia: the results of a survey of two hundred and forty-five orthopaedic surgeons. J Bone Joint Surg [Am] 1994; 76-A:1162-6.
14. Horn BD, Rettig ME. Interobserver reliability in the Gustilo and Andersen classification of open fractures. J Orthop Trauma 1993; 7:357-60.

15. Johnstone DJ, Radford WJ, Parnell EJ. Interobserver variation using the AO/ASIF classification of long bone fractures. Injury 1993; 24:163-5.

16. Andersen E, Jørgensen LG, Hededam LT. Evans' classification of trochanteric fractures: an assessment of the interobserver and intraobserver reliability. Injury 1990;21:377-8.

17. Frandsen PA, Andersen E, Madsen F, Skjødt T. Garden's classification of femoral neck fractures: an assessment of interobserver variation. J Bone Joint Surg [Br] 1988;70-B:588-90.

18. Andersen DJ, Blair WF, Steyers CM, et al. Classification of distal radius fractures: an analysis of interobserver reliability and intraobserver reproducibility. J Hand Surg [Am] 1996;21:574-82.

19. Kreder HJ, Hanel DP, McKee M, et al. Consistency of AO fracture classification for the distal radius. J Bone Joint Surg 1996;78-B: 726-31.

20. Siebenrock KA, Gerber $\mathbf{C}$. The reproducibility of classification of fractures of the proximal end of the humerus. J Bone Joint Surg [Am] 1993;75-A:1751-5.

21. Sidor ML, Zuckerman JD, Lyon $\mathbf{T}$, et al. The Neer classification system for proximal humeral fractures: an assessment of interobserver reliability and intraobserver reproducibility. J Bone Joint Surg [Am] 1993;75-A:1745-50.

22. Burstein AH. Editorial. Fracture classification systems: do they work and are they useful? J Bone Joint Surg [Am] 1993;75-A:1743-4.

23. Sjøden GOJ, Movin T, Güntner $P$, et al. Poor reproducibility of classification of proximal humeral fractures: additional CT of minor value. Acta Orthop Scand 1997;68:239-42.

24. Bernstein J, Adler LM, Blank JE, et al. Evaluation of the Neer system of classification of proximal humeral fractures with computerized tomographic scans and plain radiographs. J Bone Joint Surg [Am] 1996;78-A:1371-5. 